\title{
Morphological characterization of liquid-gas interface
}

\author{
Christophe Dumouchel*, Fabien Thiesset, Thibault Ménard \\ ${ }^{1}$ CNRS UMR 6614 CORIA, Normandy Univ., UNIROUEN, INSA Rouen, 76000 Rouen, France \\ *Corresponding author email: Christophe.Dumouchel@coria.fr
}

\begin{abstract}
The work reported in this paper focuses on the description and characterization of liquid flows in a gaseous environment such as those undergoing an atomization process. The objective here is to describe the complex forms encountered in order to study their dynamics on the basis of new indicators. Completing a work presented at ILASS-Europe 2019 (Paris), the concept of parallel surfaces is convened and extended to all scales of liquid systems. This approach leads to the identification of specific scales. By way of illustrations, this multi-scale description is applied to synthetic systems as well as to the case of the Rayleigh-Plateau instability.
\end{abstract}

\section{Keywords}

Liquid-gas interface, integral geometry, Rayleigh-Plateau instability

\section{Introduction}

Liquid atomization is a process during which the interface between the liquid and the gas varies. Most of the time, the interface sees its surface area increasing and this is precisely to take benefit from this augmentation that an amount of liquid is preferred under a spray system. Investigating liquid atomization can therefore be achieved by following the temporal evolution of the liquid-gas interface. However, the shape of the liquid systems in question is complex and is not straightforward to characterize. In this very context, three approaches have been developed: the Volume Scale Distribution (VSD) [4], the Surface-Curvature Distribution (SCD) [2] and the two-point statistics applied on the phase indicator [17].

The VSD reads as the volume comprised between the liquid-gas interface and its parallel surface at a distance $d / 2$ towards the liquid region. This parallel surface delimits the system resulting from the $d$-scale erosion of the liquid system. The scale derivative of the VSD is proportional to the surface area of the eroded system. The variations of the VSD and of the eroded system surface with the scale inform on the liquid structure characteristic scales and give access to their dynamics.

The SCD [7, 2] finds its foundation of some results of differential geometry and reads as a joint probability distribution density function of finding the mean and Gaussian curvatures at some point on the liquid-gas interface. Together with the distributions of surface area and liquid volume, a more complete set of geometrical metrics for characterizing objects of any shapes is expected with the SCD. Introducing the mean and the Gaussian curvatures allows defining the local characteristic scales of the interface as the local radius of curvature.

As suspected by [2], the VSD and SCD approaches are connected to each other. This has been mathematically established in a previous work [16] but for small scales only, i.e., those for which the demonstration may rest on differential geometry. The purpose of the present work is to extend this demonstration to any scale of the system. From a mathematical point of view, differential geometry must give way to integral geometry in order to deal with systems whose surface is no longer smooth. In this context, the morphological descriptors known as the Minkowski functionals are used because of their applicability to irregular (not smooth) surfaces.

\section{Geometrical considerations}

We consider liquid systems of any shape in a gaseous environment. Thanks to the surface tension forces, such systems always have a smooth surface. The sets of all points having 
more than one closest point on the system's boundary delineate the so-called medial axis. The minimum normal distance between the system's boundary and the medial axis is called the reach $\mathcal{R}$ of the system [8]. The medial axis and the reach constitute important morphological characteristics that vary with the deformation of the system.

To access a detailed description of the system and its evolution, we consider the series of surfaces parallel to the system surface in the liquid phase region. This series of surfaces arises from erosion of the initial system in the direction of the liquid phase, the erosion scale $d$ being twice the distance between the initial and eroded surfaces.

When $d<2 \mathcal{R}$, differential geometry allows demonstrating that the volume $V(d)$, the surface area $S(d)$, the area-weighted-average mean curvature $H(d)$ and the area-weighted-average Gaussian curvature $G(d)$ of the $d$-eroded system write [16]:

$$
\begin{aligned}
V(d) & =V(0)-S(0) \frac{d}{2}-H(0) \frac{d^{2}}{4}-G(0) \frac{d^{3}}{24} \\
S(d) & =-2 V(d)_{, d} \\
H(d) & =S(d)_{, d} \\
G(d) & =2 H(d)_{, d}
\end{aligned}
$$

where Einstein notation has been used for derivatives w.r.t $x$, i.e.

$$
\bullet_{, x}=\frac{\partial}{\partial x} \bullet
$$

These equations underline the importance of the four quantities $V(d), S(d), H(d)$ and $G(d)$ to describe the system. Theses quantities turn out to be the Minkowski Functionals (MF) of the eroded system. The MF constitute a family of morphological descriptors that describe not only the content (area) but also topology (connectivity), and shape (geometric curvature) of the system [10]. They benefit from an extensive literature dedicated to porous media, fluctuating interface, image analysis [11, 10,1]. The MF generalize curvature integrals over smooth surfaces to the case of surfaces with singular edges and corners [1] and, when combined with the morphological concept of parallel surfaces, can be used to characterize and model complex spatial structures [11].

As long as $d<2 \mathcal{R}$, Eq. (1) says that the MF's of any eroded systems are obtainable from the MF's of the initial system. When $d>2 \mathcal{R}$, this is no longer true and the system of Eq. (1) is not valid anymore. This specific case requires the help of the integral geometry to be treated and, in particular, the Steiner's formula generalizing the concept of MF's for irregular systems. When $d>2 \mathcal{R}$, we have demonstrated that Eq. (1) become:

$$
\begin{aligned}
S(d) & =-2 V(d)_{, d} \\
H(d)-l_{c} C_{2}(\theta) & =S(d)_{, d} \\
2 \pi \chi(d)+2 \pi C_{3}(\theta) & =2 H(d)_{, d}
\end{aligned}
$$

where the Euler characteristic $\chi(d)=G(d) / 2 \pi$. (Due to length restriction, the mathematical demonstration is not reproduced in this paper. It considers two systems, i.e., the eroded system at scale $d$ and the dilated system at scale $2 \epsilon$ of the eroded system at scale $d+2 \epsilon$, and compare them when $\epsilon$ approaches zero. The full demonstration will be available in the literature [6].) When $d>2 \mathcal{R}$, the eroded system presents at least one cusp, i.e., a local singularity located on the initial system medial axis at a distance $d / 2$ from the initial system boundary. According to the shape of the system, these singularities (or cusps) arrange as individual points, a line (either linear or not) or a surface. The parameter $l_{c}$ in Eqs. (3) corresponds to the length of the cusp-line. (If the cusps make a set of discontinuous points, $l_{c}=0$.) The angle $\theta$ in Eqs. (3) 

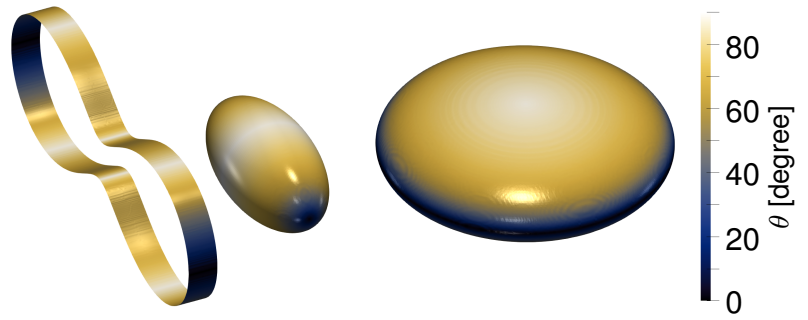

Figure 1. Representation of the different validation test cases. From left to right, the extruded Cassini oval, the prolate spheroid and the oblate spheroid. The surfaces are coloured by the angle $\theta$

is obtained from the angle $\phi$ between the normal creating the cusp and the medial axis, i.e., $\theta=\pi-\phi$. The functions $C_{2}$ and $C_{3}$ in Eqs. (3) and the lengths $l_{c}$ were established for three cases (see Table 1), i.e., extruded systems (first column), axisymmetric systems with linear medial axis (like prolate spheroids, second column) and axisymmetric systems with circular medial axis (like oblate spheroids, third column).

Table 1. Corrections $l_{c}, C_{2}$ and $C_{3}$ appearing Eqs. (3) ( $h$ is the height of the extruded system, $r_{c}$ is the radius of the medial axis)

\begin{tabular}{lccc}
\hline \hline & $\begin{array}{c}\text { Extruded } \\
\text { system }\end{array}$ & $\begin{array}{c}\text { Axisym. system } \\
\text { lin. axis }\end{array}$ & $\begin{array}{c}\text { Axisym. system } \\
\text { circ. axis }\end{array}$ \\
\hline$l_{c}$ & $h$ & 0 & $2 \pi r_{c}$ \\
$C_{2}(\theta)$ & $\tan \theta-\theta$ & - & $\tan \theta-\theta$ \\
$C_{3}(\theta)$ & 0 & $\frac{(1-\cos \theta)^{2}}{\cos \theta}$ & $\frac{\theta}{\cos \theta}-\sin (\theta)$ \\
\hline \hline
\end{tabular}

\section{Numerical Validation}

We start by validating Eq. (3) using synthetic shapes such as those shown in Fig.1, i.e., the extruded Cassini oval, the prolate spheroid and the oblate spheroid. In all situations, an approximation of the level-set function is first initialized before proceeding to the re-initialization algorithm of [14] to ensure that the different level-set form parallel surfaces. For this purpose, we use the ARCHER code $[12,18]$. The volume $V(d)$, surface area $S(d)$, mean $H(d)$ and Gaussian curvatures $G(d)$ for each level-set $d / 2$ are computed using the method of $[7,3]$ which has the main advantage of incorporating a topological constraint in the curvature calculation (the Gauss-Bonnet theorem). We use prior versions of the routines now available through the Mercur(v)e project *.

To obtain the set of points of the medial axis, we compute for each vertex on the triangulated zero level-set surface, the location of the intersection between the normal issuing from this vertex and either the $x z$-plane for extruded systems, the $x$ axis for axisymmetric objects with linear medial axis or $x y$-plane for axisymmetric objects with circular medial axis. Then, the angle $\theta$ and distance from the medial axis can be readily computed using trivial trigonometry. For each scale $d / 2$, we sum the contribution $l_{c} C_{2}, C_{3}$ of all vertex whose distance from the medial axis falls within an interval $d / 2-\Delta d / 2: d / 2+\Delta d / 2$. $\Delta d$ was chosen sufficiently small for not loosing some scale dynamics and sufficiently large to ensure statistical convergence. Generally, 150 to 200 points for the scale parameter $d / 2$ were prescribed, ranging from zero to the maximum erosion scale (i.e. the scale beyond which the system is fully eroded).

${ }^{*}$ http://docs.mercurve.rdb.is/ 


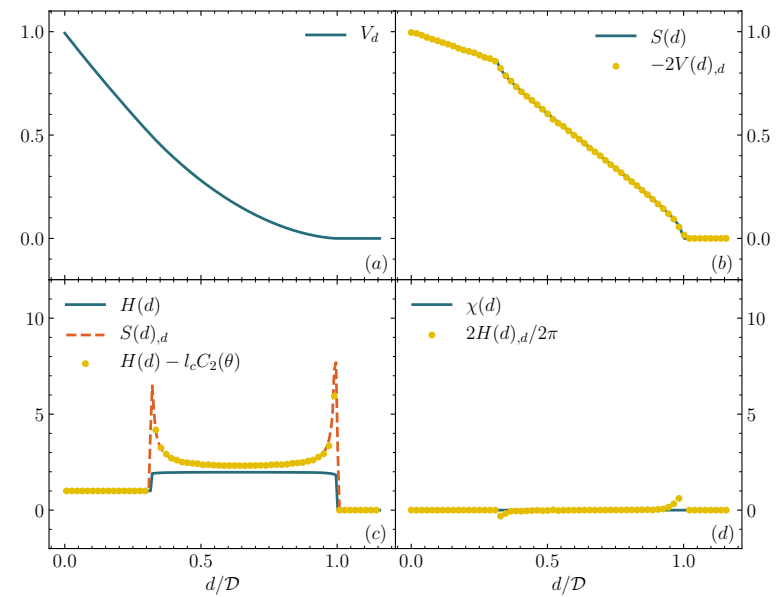

Figure 2. (a) Volume $V(d)$, (b) surface area $S(d)$, (c) mean curvature $H(d)$ and (d) Euler characteristic $\chi(d)$ as a function of the erosion scale $d$ for the extruded Cassini oval.

\section{The extruded Cassini oval}

The Cassini oval is parametrized with the location of the foci $a$ aligned with the $x$ axis, and the shape parameter $b$ which were chosen in such way that $b / a=150 / 148$. The scale $d$ which ought to be reached for the Cassini oval to be fully eroded is the size of the bulge and is equal to $\mathcal{D}=b^{2} / 2 a$. The reach for such a parametrization of the Cassini oval is equal to half the size of the neck and is given by $\mathcal{R}=\left(b^{2}-a^{2}\right)^{1 / 2}$. Hence, we then expect the correction $l_{c} C_{2}$ (see Eq. 3) to be non-zero for scales $d \gtrsim 0.32 \mathcal{D}$.

Figure 2 depicts the functions $V(d), S(d), H(d)$ and $\chi(d)$ for the Cassini oval. (The scale $d$ is normalized by $\mathcal{D}=2 b$ and the functions (except $\chi(d)$ ) are normalized by their respective values at $d=0$.) We observe that the function $H(d)$ has to be corrected to reproduce the function $S(d)_{, d}$. As expected, the correction starts at the scale $d \approx 0.32 \mathcal{D}$. At this scale, $S(d){ }_{, d}$ shows a sudden peak followed by a sharp decrease. This behavior, nicely reproduced by the correction $l_{c} C_{2}$, is a characteristic of a cusp due to a throat or bottleneck. The scale at which this first peak appears is therefore equal to the diameter of the Cassini oval bottleneck. At $d \approx 0.65 \mathcal{D}$, $S(d)_{, d}$ starts a continuous increase that ends as a peak at the maximum scale $\mathcal{D}$. This behavior is well reproduced by the function $l_{c} C_{2}$ given in Eqs.(3) and in Table 1. This second peak is imposed by the bulge of the oval and occurs at the maximum bulge scale which appears to be the one of the system also. We see here that the peak shapes of the function $S(d)_{, d}$ allow dissociating bottleneck and bulge characteristic scales. The value of $H(d)$ between the two peaks indicates that the eroded system becomes the union of two disconnected systems for this range of scales.

\section{Validation on axisymmetric systems}

Numerical validations were also performed for axisymmetric systems such as a prolate and an oblate spheroid (see Fig. 1). The medial axis of these systems is linear and circular, respectively. Equations (3) together with the expressions of the length $l_{c}$ and of the functions $C_{i}(\theta)$ given in Table 1 (second column for the prolate spheroid and third column for the oblate spheroid) succeeded in both cases to connect the MF's of the eroded systems, without any restriction on the value of the erosion scale $d$.

As expected, for the prolate system no correction of $H(d)$ was necessary to reproduce $S(d)_{, d}$ since $l_{c}=0$. However, for the oblate system, $H(d)$ requires a correction proportional to the circumference of the medial axis as indicated in Table 1. For both systems, a correction of $\chi(d)$ was necessary to reproduce the scale derivative $H(d)_{, d}$. (The detailed results for these cases, as well as for others, will be shortly available in [6].) 


\section{Application to multiphase flows}

The analysis is now applied to real systems. Here we focus on the ability of parallel sets to provide insights into the morphology of deformed liquid structures evolving in a gaseous atmosphere in the case of a cylindrical liquid ligament submitted to the classical Plateau-Rayleigh instability.

\section{Description of the ARCHER code}

Data of liquid/gas flows are gathered using the High-Performance-Computing code ARCHER developed at the CORIA laboratory [12]. It is based on the one-fluid formulation of the incompressible Navier-Stokes equation which is solved on a Cartesian mesh, viz.

$$
\partial_{t} \rho \boldsymbol{u}+\boldsymbol{\nabla} \cdot(\rho \boldsymbol{u} \otimes \boldsymbol{u})=-\boldsymbol{\nabla} p+\boldsymbol{\nabla} \cdot(2 \mu \boldsymbol{D})+\boldsymbol{f}+\gamma \mathcal{H} \delta_{s} \boldsymbol{n} .
$$

$p$ is the pressure field, $\boldsymbol{D}$ the strain rate tensor, $\boldsymbol{f}$ a source term, $\mu$ the kinematic viscosity, $\rho$ the density, $\gamma$ the surface tension, $\boldsymbol{n}$ the unit normal vector to the liquid-gas interface, $\mathcal{H}$ its mean curvature and $\delta_{s}$ is the Dirac function characterizing the location of the liquid gas interface. For solving Eq. (4), the convective term is written in conservative form and solved using the improved Rudman technique [13] presented in [18]. The method of [15] is used to compute the viscous term. To ensure incompressibility of the velocity field, a Poisson equation is solved. The latter accounts for the surface tension force and is solved using a MultiGrid preconditioned Conjugate Gradient algorithm (MGCG) [19] coupled with a Ghost-Fluid method [9].

A coupled level-set and volume-of-fluid (CLSVOF) solver is used for transporting the interface, the level-set function accurately describing the geometric features of the interface (its normal and curvature) and the volume-of-fluid function ensuring mass conservation. The density is calculated from the volume-of-fluid (or liquid volume fraction) as $\rho=\rho_{l} \phi+\rho_{g}(1-\phi)$, where $\rho_{l}, \rho_{g}$ is the density of the liquid and gas phase. The dynamic viscosity $\left(\mu_{l}\right.$ or $\left.\mu_{g}\right)$ depends on the sign of the Level Set function. In cells containing both a liquid and gas phase, a specific treatment is performed to evaluate the dynamic viscosity, following the procedure of [15]. For more information about the ARCHER solver, the reader can refer to e.g. $[12,18]$. The volume $V(d)$, surface area $S(d)$, mean $H(d)$ and Gaussian curvatures $G(d)$ for each level-set $d / 2$ are computed using the method of [3].

\section{The Plateau-Rayleigh instability}

This section presents the case of a cylindrical ligament subject to the surface tension driven Rayleigh-Plateau instability.

We employ the same numerical configuration as [2]. The liquid and gas density are $\rho_{L}=1000$ kg.m $\mathrm{m}^{-3}$ and $\rho_{G}=1 \mathrm{~kg} \cdot \mathrm{m}^{-3}$, respectively. The viscosity of the liquid phase is $\mu_{L}=110^{-3}$ $\mathrm{kg} \cdot \mathrm{m}^{-1} \cdot \mathrm{s}^{-1}$, and that of the gas phase $\mu_{G}=1.87910^{-5} \mathrm{~kg} \cdot \mathrm{m}^{-1} \cdot \mathrm{s}^{-1}$. The surface tension $\gamma=0.072 \mathrm{~kg} . \mathrm{s}^{-2}$. The ligament has an initial radius $a=\bar{k} L_{x} / \pi$ where the non-dimensional perturbation wavenumber $\bar{k}=k a=0.55$ applies on the $x$ axis. The amplitude of the perturbation was set to $0.1 a$. Only a quarter (half) of the ligament is simulated in the azimuthal (streamwize) direction. The computational domain is $L_{x}=3 L_{y} / 2=3 L_{z} / 2=1.510^{-4} \mathrm{~m}$ and $192 \times 128 \times 128$ grid points are used in $x, y$ and $z$ directions, respectively.

For the selected sinusoidal perturbation wave-number, the cylindrical ligament is unstable and, according to the Rayleigh theory, the perturbation temporal growth rate is equal to $\omega=$ $0.318 \tau_{\gamma} /(8)^{1 / 2}$ (where $\tau_{\gamma}=0.045 \mathrm{~ms}$ ) and the break-up time is $t_{b u}=\tau_{\gamma} \log \left(D / 2 \eta_{0}\right) /\left(\omega\left(8^{1 / 2}\right)\right)$, i.e., $\tau_{b u}=0.115 \mathrm{~ms}$. The deformation of the ligament manifests first by a growth of the amplitude of the sinusoidal perturbation and, second, by a rearrangement of the ligament as an almost spherical bead connected with a small scale ligament. The rupture of this structure produces one big drop (from the bead) and one satellite drop, the latter coming from the relaxation of the small ligament when left alone after the break-up.

Figure 3 presents the functions $V(d), S(d), H(d)$ and $\chi(d)$ for the three cases, i.e., during the initial sinusoidal perturbation growing (case (1)), just before the break-up event (case (2)) and 


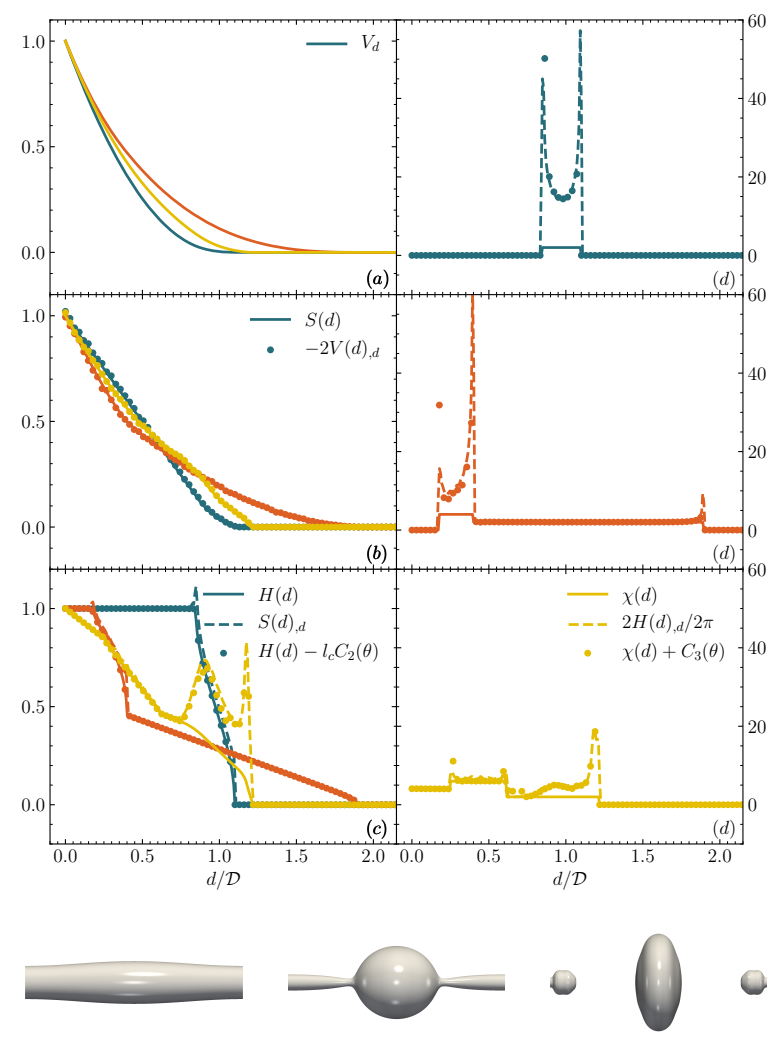

Figure 3. (a) Volume $V(d)$, (b) surface area $S(d)$, (c) mean curvature $H(d)$ and (d) Euler characteristic $\chi(d)$ as a function of the erosion scale $d$ for a liquid ligament undergoing the Plateau-Rayleigh instability. Results are for $t / \tau_{\text {bu }}=$ (blue) 0.42 , (red) 0.96, (yellow) 1.09 .

after the break-up event (case (3)). As for the previous situations, we note that the functions $S(d)$ and $V(d)_{, d}$ remain proportional for the three cases (Fig. 3(b)). As expected, the functions $H(d)$ and $S(d)_{, d}$ match well for cases (1) and (2), i.e., those having a linear medial axis and requiring no correction at this level. Since case (3) shows an quasi-oblate element, a correction of $H(d)$ appears necessary to retrieve $S(d)_{, d}$. Once again we note that the correction provided by Eq. (3) apply very well.

Since the system remains homeomorphic to a cylinder before break-up, the Euler characteristic $\chi(0)$ is equal to zero for cases (1) and (2) (see Fig. 3(d)). However, for other scales, $\chi(d)$ varies according to the number of elements constituting the eroded system. This number increases when the erosion scale matches the diameter of a bottleneck or a pinch-off, or decreases when the erosion scale matches the diameter of a swell section. The scales concerned by this Euler characteristic change are rendered visible by the peaks of the function $H(d)_{,} d$. (Note that, here again, Eq. (3) and the elements of Table 1 combine very well and ensure a good representation of the function $\left.H(d)_{, d}\right)$. Referring to the observations made in Fig. 2, the first peak for case (1) indicates the scale of a pinch-off and the second one the scale of a bulge. These two scales correspond to the smaller and larger diameters of the deformed ligament, respectively. For case (2), the first peak indicates the diameter of the pinch-off between the ligament and the bead, the second peak indicates the larger diameter of the ligament and the third peak is the characteristic scale of the bead. For case (3), $\chi(0)=4$ since the system has transformed as two drops, the small one looking like a peanut. This value changes as a function of the scale indicating that these drops are not fully spherical yet.

Figure 4 displays the temporal evolution of three specific scales: $D_{\min }, D_{1 \max }$ and $D_{2 \max }$, the time being normalized by the theoretical break-up time $\tau_{b u}$. These scales are those at which $H(d)_{, d}$ exhibits a peak with $D_{\min }<D_{2 \max }<D_{1 \max }$. Being the smallest scale for which a correction of $\chi(d)$ is required, $D_{\min }$ is equal to $2 \mathcal{R}$. And the fact that the reach of the system is associated with a peak of the function $H(d)_{, d}$ means that it always corresponds to the radius of 


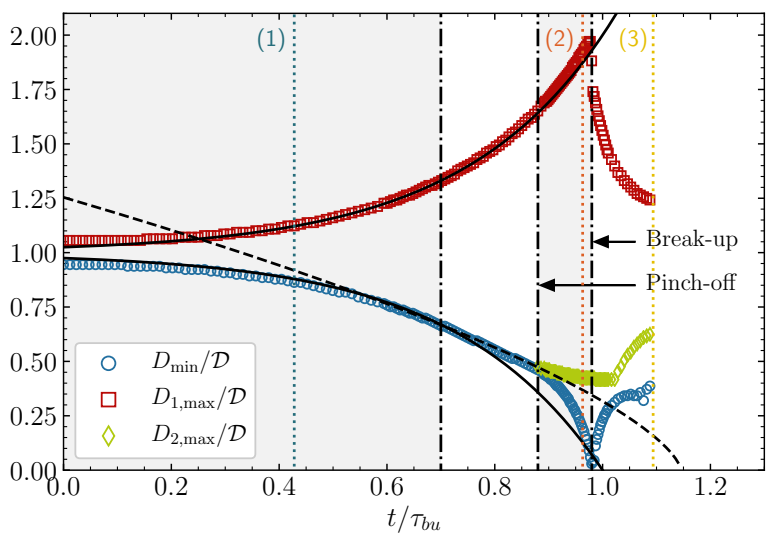

Figure 4. Time evolution of the reach $D_{\min }$, the maximum erosion scale $D_{1 \text {, max }}$ and the size of the satelite maximum erosion scale $D_{2, \max }$ for the Plateau-Rayleigh instability. The two vertical black lines depict the first departure of $D_{\min }$ from an exponential behavior and the apparition of the pinch-off, respectively. The three time instant labelled (1) $t=0.42 \tau_{b u}$, (2) $t=0.96 \tau_{b u}$ and (3) $t=1.09 \tau_{b u}$ are also displayed by vertical dotted lines

a bottleneck or of a pinch-off.

The unstable nature of this system is manifested by a marked divergence of the scales $D_{\min }$ and $D_{1 \text { max }}$. The scale $D_{1 \max }$ continuously increases following very well the exponential growth obtained from the linear theory and shown by the black line. Similarly, the scale $D_{\min }$ reports a continuous decrease that follows the theoretical behavior up to $t / \tau_{b u}=0.7$. After this time, the linear theory is not appropriate anymore to reproduce the evolution of $D_{\min }$ which, therefore, is likely affected by the first action of non-linear effects. The black dash line in Fig. 4 shows that $D_{\min }$ decreases as $t^{2 / 3}$. As reported by the literature ([5] for instance) this temporal dependence reveals that the pinch-off contraction has reached an inertial regime for which the dominant resistance against surface tension stems from the inertia of accelerating fluid elements. This regime lasts until $t / \tau_{b u}=0.9$ after which another dynamics appears. This last behavior likely represents the visco-capillary regime for which the dominant resistance against surface tension stems from viscous forces. The theory predicts a linear dependence between the pinch-off diameter and the time for this regime that is not obvious in Fig. 4 although the points seem to align close to the break-up event. At this very time $\left(t / \tau_{b u}=0.9\right)$, a third peak of $H(d)_{, d}$ appears at scale $D_{2 \text { max }}$. The appearance of this scale demonstrates that the pinch-off evolution divides the liquid system in two isolated parts, confirming the approach of a breakup event. Each of these parts have their own maximum scale, $D_{2 \max }$ being the one of the ligament. We see that during break-up, this scale does not vary significantly.

The succession of the three $D_{\text {min }}$ decay regimes identified in Fig. 4 agrees in a negative second time derivative just before the break-up event. We see in this last example that the multiplication of $H(d)_{, d}$ peaks also indicates the imminence of break-up.

\section{Conclusions}

In this paper, the connections between the MF's of parallel systems obtained by erosion have been established for the whole initial system scale range. As far as liquid atomization process is concerned, this description technique provides interesting results. For instance, the first information of interest is the fact that a system having a reach less than the maximum scale denotes a liquid system not in equilibrium with a shape that changes over time. When the erosion is greater than twice the reach, the MFs are not simply related to their successive derivatives and depend on local properties of the interface. This dependence of the MFs is highly a function of the shape of the system medial axis. Furthermore, it allows identifying specific scales such as those for which a bottleneck or a pinch-off appears. As done for the case of the Rayleigh-Plateau, exploring the dynamics of these scales appears a good way to detect the imminence of break-up events. 


\section{Nomenclature}

$d \quad$ Scale $[\mathrm{m}]$

$G(d) \quad$ Area-weighted-average Gaussian curvature of $d$-eroded system [-]

$H(d) \quad$ Area-weighted-average mean curvature of $d$-eroded system [m]

$S(d) \quad$ Area of $d$-eroded system [ $\left.\mathrm{m}^{2}\right]$

$V(d) \quad$ Volume of $d$-eroded system $\left[\mathrm{m}^{3}\right]$

$\mathcal{R} \quad$ Reach of a system [m]

$\chi(d) \quad$ Euler characteristic of $d$-eroded system [-]

\section{References}

[1] Arns, C.H., Knackstedt, A., Mecke, R., 2004, Colloids and Surfaces A; Physicochemical and Engineering Aspects, 241(1-3) pp. 351-372.

[2] Canu, R., Puggelli, S., Essadki, M., Duret, B., Massot, M., Reveillon, J., Demoulin, F.X., 2018, Int. J. Multiphase Flow, 107, pp. 230-245.

[3] Di Battista, R., Bermejo-Morena, I., Ménard, T., de Chaisemartin, S., Massot, M., 2019, 10th International Conference on Multiphase Flow, Rio de Janeo, Brazil.

[4] Dumouchel, C., July. 30.-Aug. 3. 2017, ASME-FEDSM, FEDSM2017-69590.

[5] Dumouchel, C., Aniszewski, W., Vu, T.T., Ménard, T., 2017, Int. J. Multiphase Flow, 92, pp. 181-192.

[6] Dumouchel, C., Thiesset, F., Ménard, T., 2021, under review.

[7] Essadki, M., Drui, F., Larat, A., Ménard, T., Massot, M., 2019, Int. J. Multiphase Flow, 120:103084.

[8] Federer, H., 1959, Transactions of the American Mathematical Society, 93(3), pp. 418-491.

[9] Fedkiw, R.P., Aslam, T., Merriman, B. Osher, S., 1999, J. Comput. Phys., 152(2), pp. 457492.

[10] Mantz, H., Jacobs, K., Mecke, K., 2008, Journal of statistical Mechanics: Theory and Experiment, (12), p. 12015.

[11] Mecke, K., 2002, Physica A: Statistical Mechanics and its Applications, 314(1-4), pp. 655662.

[12] Menard, T., Tanguy, S., Berlemont, A., 2007, Int. J. Multiphase Flow 33(5), pp. 510-524.

[13] Rudman, M., 1998, Int. J. Number Methods Fluids, 28(2), pp. 357-378.

[14] Sussman, M., Smereka, P., Osher, S., 1994, , 2005,J. Comput. Phys., 114(1), pp. 146-159.

[15] Sussman, M., Smith, K.M., Hussaini, M.Y., Ohta, M., Zhi-Wei, R., 2007, J. Comput. Phys., 221(2) pp. 469-505.

[16] Thiesset, F., Dumouchel, C., Ménard, T., Aniszewski, W., Vaudor, G., Berlemont, A.,2019, ILASS-Europe, September 2-4, 2019, Paris (France)

[17] Thiesset, F., Duret, B., Ménard, T., Dumouchel, C., Reveillon, J., Demoulin, F.X., 2020, , JFM, 886, pp. A4-1:A4-32.

[18] Vaudor, G., Ménard, T., Aniszewski, W., Doring, M., Berlemont, A., 2017, Comp. Fluids, 152, pp. 204-216.

[19] Zhang, J., 1996, Appl. Math. Comput., 80(1) pp. 73-93. 